\title{
Most representative parameters of voltage dips.
}

\author{
T. Yebra, V. Fuster, A. Quijano, M. García. \\ Instituto de tecnología eléctrica \\ Avda. Juan de la Cierva, 24, Parque Tecnológico de Valencia, 46980 Paterna (Valencia) \\ Edificio Institutos 2, Universidad Politécnica de Valencia. Camino de Vera s/n, 46022 Valencia \\ Telf.: +34 961366670 Fax.: +34 961366680 e-mails: ite@itenergia.com, \\ tomas.yebra@ite.es, vicente.fuster@ite.es, alfredo.quijano@ite.es, marta.garcia@ite.es.
}

\begin{abstract}
This paper deals with the aspects that allow measurement systems to identify and characterize voltage dips. The objective has been to select the most representative parameters of voltage dip for later analysis. The selection has been made on the basis of real registers in low voltage networks. Several algorithms have been implemented to obtain these parameters. In each case, at least two different algorithms have been used to measure the same parameter. This methodology has been allowed to compare the results and their accuracy.
\end{abstract}

Key words: Voltage dips, voltage measurement, characterization of voltage dips, power quality.

\section{Introduction}

Studies of voltage dips have the common objective of the description of the physical phenomenon from different points of view. Some papers deal with the effects caused by dips in the facilities, their parameters and the frequency of apparition, the severity or their influence in different type of loads [1 - 4]. Others researches approach the problem with the maintenance of the electrical facilities. They stressed the importance in the origin, the causes or their propagation through the power system [5 8]. Another third group approach voltage dips as phenomenon to eliminate. They give importance to the energy no supplied, the study detection systems of voltage dips or the response of the systems of uninterrupted power supply with different technologies [9 - 10]. Of course this classification is not rigorous and there are more points of view [11 - 14] that are examples of current researches. However all of them have in common the search of measured magnitudes that are able to characterize the voltage dip in a suitable form for the study that it being carried out.

In the present paper, it has been studied the measure method for the most noticeable parameters, suitable for the studies about voltage dips mentioned in the previous paragraph. First, a parameter selection has been performed, based on their physical sense and taking into account the advantages and disadvantages that can be found measuring the parameters in a real voltage dip register.

The selected parameters have been defined univocally and the measurement algorithm has been implemented to estimate these parameters. It has been implemented the measure with different calculations. In order to measure the parameter it has been considered the use of different reference waves. That it let to make a comparison of the measure of the parameter, moreover to conclude about what algorithm o reference wave is better for charactering the parameter of the dip from the physical point of view.

The adoption of the algorithms has followed two approaches. The normative approach considers the recommendations of different organizations in questions about the electrical measurements and electromagnetic compatibility. The second approach follows some papers results about dip measurements [15 - 20]. This paper determinates what methods of measure are more appropriated according to the objectives and the problems that can appear.

The objective thus, it may be chosen the most appropriated algorithm, in function of the degree of accuracy in the measurement of the dip, which will be more or less relevant according the type of study.

\section{Magnitude measurement}

The measure of the magnitude can be done by different ways in depending on the type of algorithm that it is used to evaluated and the reference that is taken as limit value when it is given a percentage values.

The most estimation methods of magnitude value are based on all data of the sampled signal. There are others alternatives like the use of the peak of the voltage in a period or the RMS value of the fundamental component. In some cases, these alternatives may be valid, but the physical meaning of RMS voltage, related to power calculus, makes it more suitable for the characterization of the magnitude of voltage dips.

For continuous signals, the expression of RMS is:

$$
V_{r m s}=\sqrt{\frac{1}{T} \int_{t}^{t+T} v^{2}(\tau) d \tau}
$$

The discretization of equation (1) is: 


$$
V_{r m s}=\sqrt{\frac{1}{N} \sum_{i=1}^{N} v_{i}^{2}}
$$

Where:

$\mathrm{N}=$ number of samples in the integration period.

$v_{\mathrm{i}}=$ are the samples in the time domain.

\section{A. Calculus with a fixed sample window}

The registers are digital. They sample the voltage and calculate the RMS voltage using discretization algorithms. Two of this group has been selected as more representative.

According to IEC 6100-4-30 [21], the expression (2) must be integrated in one cycle. If it is used a window more large it would miss information about the change of the signal due to the inclusion of values that they make soft the signal and reduce the absolute value of the variation. A sorter window of half cycle is not used because it introduces fictitious oscillations with twice the fundamental frequency. This effect is avoided if the window is multiple integer of half cycle of the fundamental frequency.

The equation (2) is transformed in:

$$
V_{r m s}(N)=\sqrt{\frac{1}{N} \sum_{i=(k-1) N+1}^{i=k N} v_{i}^{2}}
$$

Signal with constant amplitude, the RMS is independent of the phase-angle at the beginning of the calculus. But it is not the case when there is a change in the amplitude, like in voltage dips.

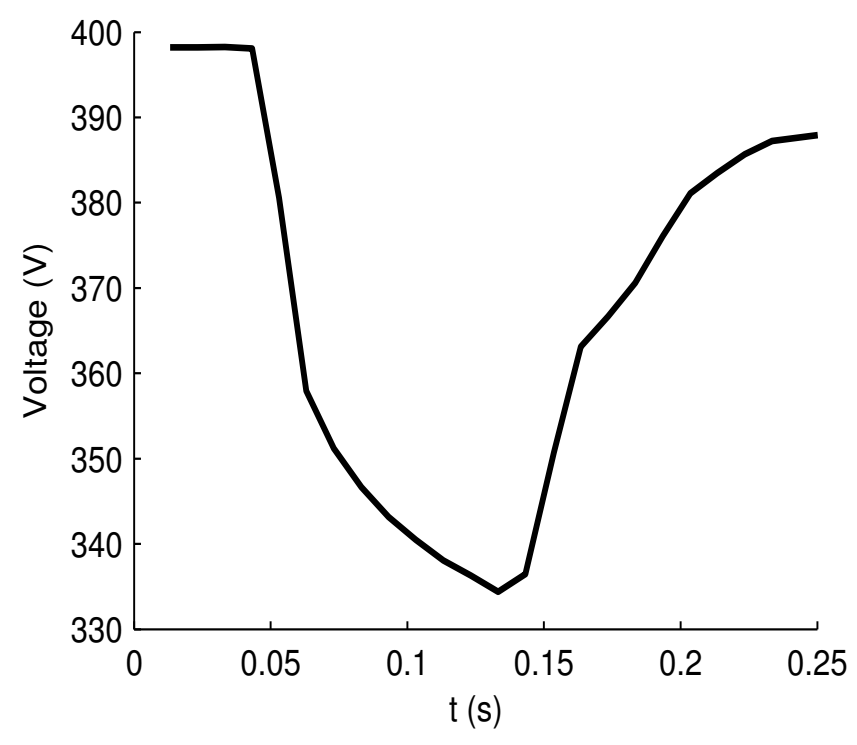

Fig. 1. RMS voltage dip signal calculated according to IEC 6100 - 4 - 30

The register equipment samples the signal with a window of one cycle commencing zero crossing and refreshed each half cycle. Then, two consecutive RMS measurements have $\mathrm{N} / 2$ samples in common.

The figure 1 shows the wave form of a voltage dip when it is applied the RMS algorithm which flowchart is shown in figure 2 .

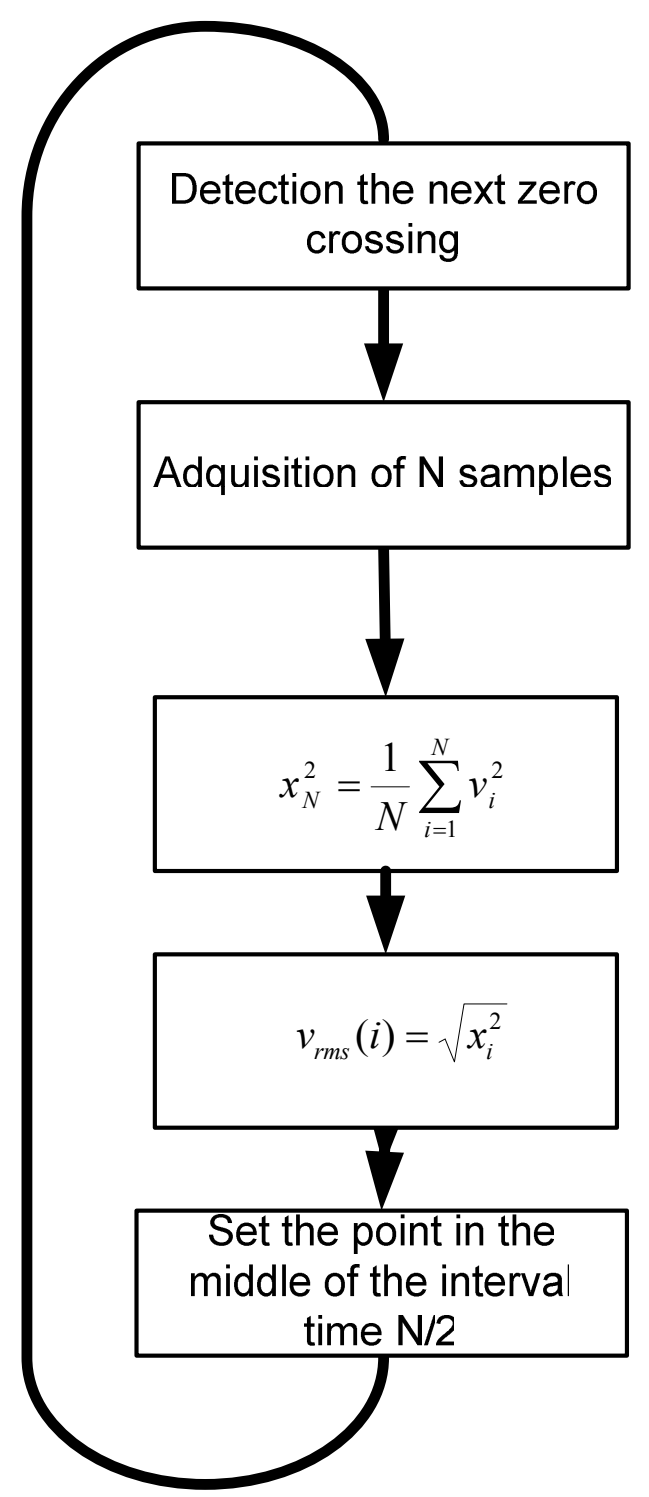

Fig. 2. Algorithm of calculus of RMS with a window of one cycle and refreshes each half cycle.

\section{B. One sample mobile window}

It is possible to refresh the RMS value integration window with a step of one sample, obtaining more continuous RMS signal of a voltage dip.

The disadvantage of this method is that produces oscillations, because it is more dependent on waveform changes, so and as mentioned before. On the other hand it detects faster the amplitude changes in the original voltage.

The use a fixed sample window method there is not optimal from the point of view computational, since a signal of period $\mathrm{N}$ samples that has to refresh each 
sample it has N-1 samples repeated in the estimation of the RMS value. It is possible to get a fast calculus thought a mobile window.

Figure 3 shows the flowchart of the algorithm. $\mathrm{N}$ is the number of samples in one period of the signal. The first $\mathrm{N}$ samples are obtained and it is calculated the sum of square values.

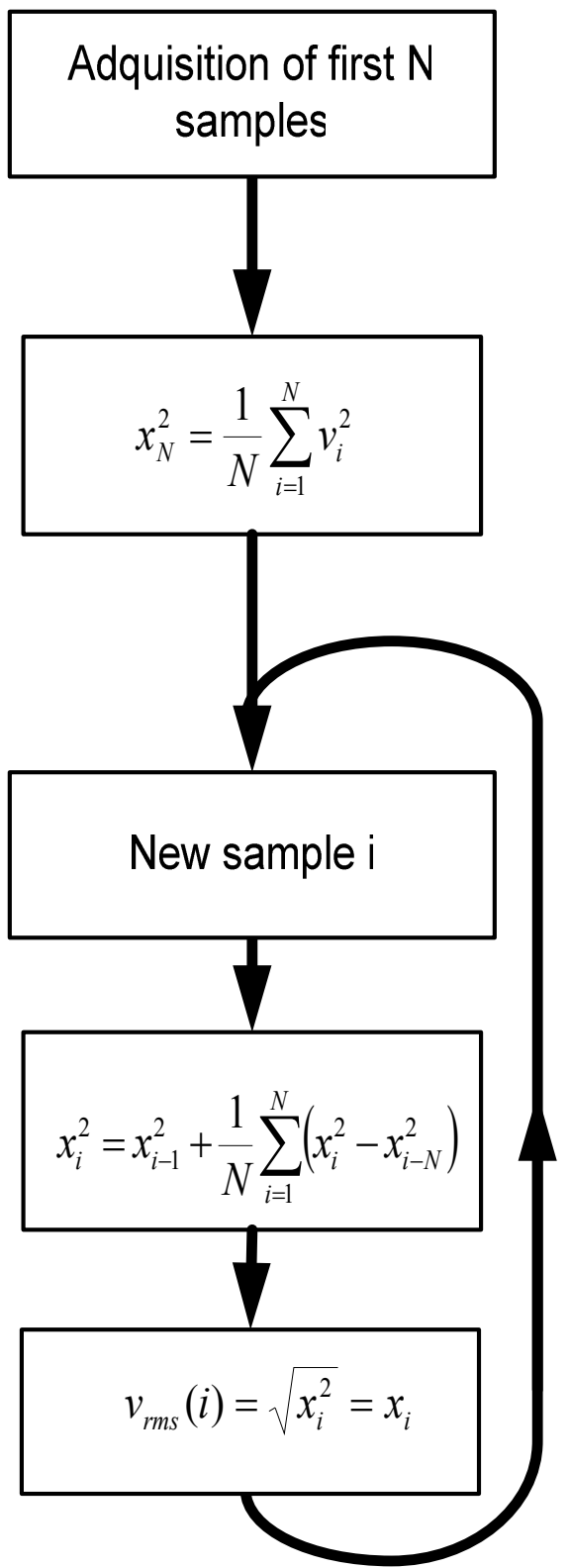

Fig.3. Algorithm of calculus of RMS. Iterative method.

At the moment $\mathrm{k}+\mathrm{N}-1$, the square $\mathrm{RMS}$ value it is obtained by means of the expression:

$$
x_{k+N-1}^{2}=\frac{1}{N} \sum_{i=k}^{k+N-1} v_{i}^{2}
$$

At the moment $\mathrm{K}+\mathrm{N}$, the square RMS it will be.

$$
x_{k+N}^{2}=\frac{1}{N} \sum_{i=k+1}^{k+N} v_{i}^{2}
$$

The equation (5) can be written as iterative form using $x_{k+N-1}^{2}$ in the next form.

$$
x_{k+N}^{2}=\frac{1}{N} \sum_{i=k+1}^{k+N} v_{i}^{2}=\frac{1}{N}\left(-v_{k}^{2}+N x_{k+N-1}^{2}+x_{k+N}^{2}\right)
$$

The result is:

$$
x_{i}^{2}=x_{i-1}^{2}+\frac{1}{N}\left(-v_{i-N}^{2}+v_{i}^{2}\right)
$$

The figure 4 shows real signal processed by this algorithm.

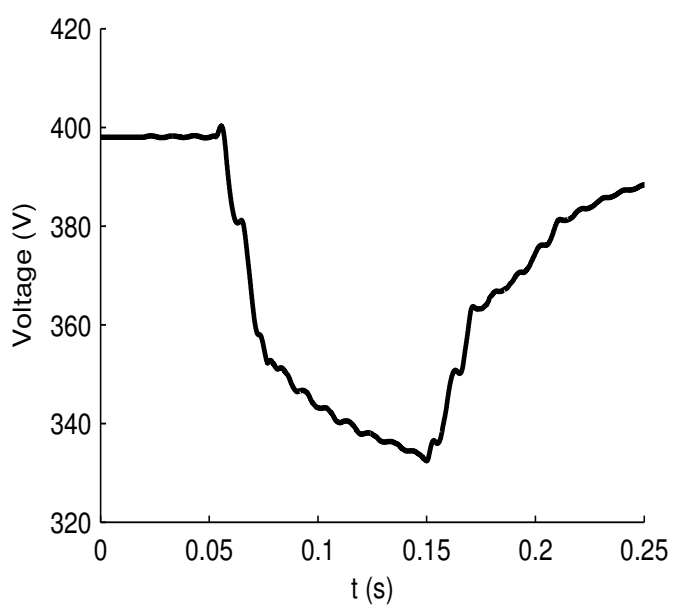

Fig. 4. RMS signal with a voltage dip when it is calculated with iterative method.

The method is developed adding the square value of the next sample and subtracting the square value of the first sample. Once the new value of $x_{i}^{2}$ is obtained it is applied the square root. More detailed explanation can be found in [22].

\section{Parameters related to magnitude}

Once it has been obtained the RMS curve of the voltage dip. It can be defined the parameters related with the magnitude. Most representative are:

Pre-dip voltage: RMS value of voltage before the beginning of the dip. It must be understood the initial moment of the dip as the moment when the RMS voltage crosses down an established level.

Post-dip voltage: RMS value of voltage after the end of the dip. It must be understood the final moment of the dip as the moment when the RMS voltage crosses up an established level.

Depth of dip: Maximum percentage of the voltage that it is not supplied during the dip. The depth is related to one reference voltage level (nominal voltage, pre-dip voltage, declared voltage...).

Remaining voltage: Percentage of voltage remains while lasted the dip. It is related to one reference voltage level. 
Mean of the dip: Average of the values of RMS voltages between the beginning and the end of the dip.

Area of dip: Value of the area locked up between the RMS pre-dip voltage and the RMS signal in the interval that includes the dip. This value matches with the subtraction of two integrals, the integral of the straight line of pre-dip voltage minus the integral of actual voltage in the interval that defines the duration of the dip.

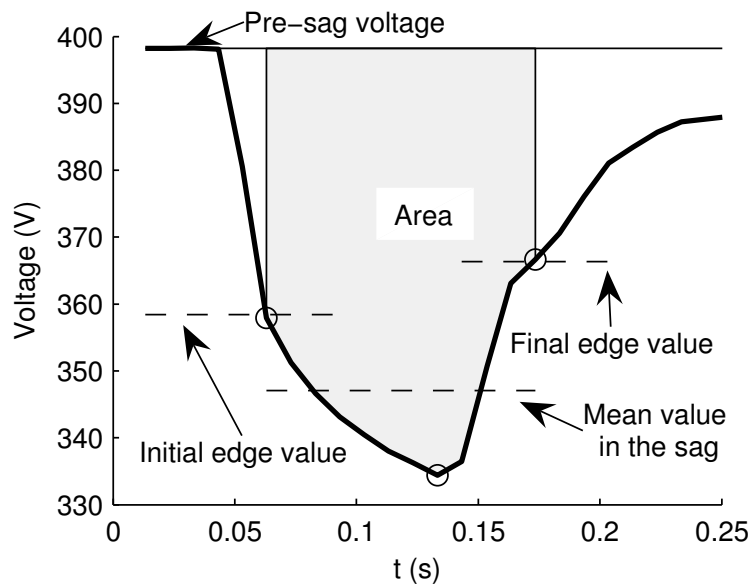

Fig.5 Voltage dip calculated with the block method and its parameters related to the magnitude.

Figures 5 and 6 show the parameters defined. Figure 5 uses RMS wave calculus with a fixed sample window. Figure 6 uses RMS wave with one sample mobile window.

Most usually parameters used in the disturbance analysis are the depth and the remaining voltage. Nevertheless there is not agreement on the level of reference voltage that it may be chosen to the percentage values.

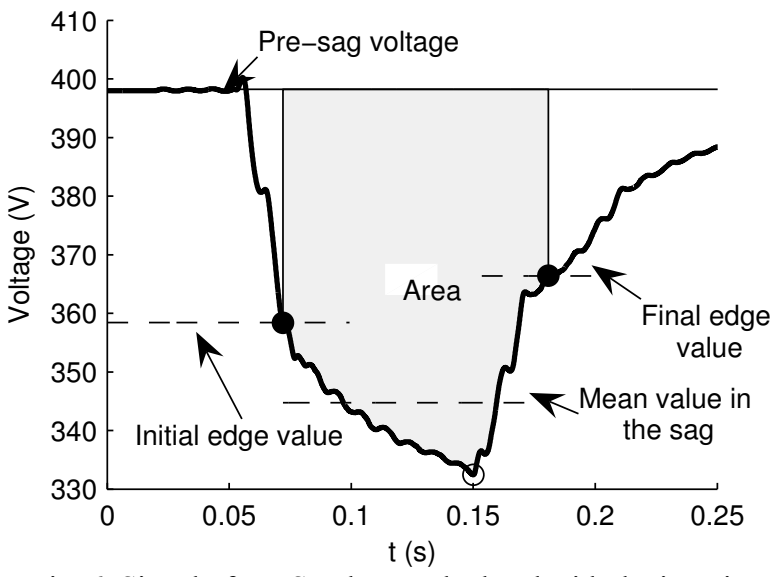

Fig. 6. Signal of RMS voltage calculated with the iterative method and its parameters related to the magnitude.

One argument in favour of using of nominal voltage as reference is because it is constant, but it has the disadvantage that it has not good results when the real voltage is higher or lower than the nominal voltage. That reduces or increases the magnitude of the dip. On the other hand the depth gives a more clear idea of the severity of the disturbance than the remaining voltage.

The remaining voltage has the disadvantage that gives a value bigger to the disturbances lesser hard. A dip of $100 \%$ means that there is not dip. Otherwise it is more clear the evaluation of the magnitude because it is known what the voltage value is just calculating a multiply by the nominal voltage that is a reference common.

The mean value and the area have not these disadvantages. The mean value has the problem that can hide the severity of the dip if the fall and the recovery of RMS voltage are fast. The area is a good indicator of the severity of the phenomenon but it has the disadvantage that is not a direct measure because there are many magnitudes that take part on it and increase the uncertainty of the measure.

It is recommendable to use the depth or the remaining voltage if the main objective is to register the variation of the voltage, because these magnitudes are more related to the range of the voltage. On the other hand if the main objective is to get an idea of severity it is recommendable to use the measure of area because it has take into account the time implicitly.

\section{Phase-angle measurement}

Another physical measurement that can be taken in the phasor of voltage is the temporal evolution of the phaseangle. Generally the evaluation of this measurement needs sophisticated mathematical tools as FFT or Wavelet transforms. It is proposed others alternative algorithms that calculated the evolution of phase-angle at the moments more interesting to analyze in the voltage dips.

The main cause of interest in this parameter is the influence in the synchronization of electronic devices. During the dip, a variation of the phase-angle and frequency is produced. This moves the crossing by zero that most of semiconductors take as reference in their commutation. The variation of the crossing by zero can produce the collapse of the control machine.

\section{A. Algorithms of phase-angle measurement}

1) Instantaneous phase-angle estimation algorithm.

This algorithm calculates the temporal evolution of the phase-angle during dip; it was proposed by H.-S. Song and K. Nam [23 - 24]. The main advantage is its speed, so that it is not used the Fourier transform.

The algorithm is based on the use of WLSE method (weighted least-squares estimation).

A voltage signal can be discomposed as:

$$
E(t)=\bar{E} \cos (\omega t+\phi)=E_{d} \cos (\omega t)-E_{q} \sin (\omega t)
$$


$\bar{E}$ is the amplitude, $\omega$ is the pulsation and $\Phi$ the angle.

$$
\begin{aligned}
& E_{d}=\bar{E} \cos (\Phi) \\
& E_{q}=\bar{E} \sin (\Phi)
\end{aligned}
$$

At steady state $\mathrm{E}_{\mathrm{d}}, \mathrm{E}_{\mathrm{q}}$ and $\Phi$ are constants. The expression can be written as:

$$
y\left(t_{i}\right)=H\left(t_{i}\right) x\left(t_{i}\right)
$$

Where

$$
\begin{aligned}
& H\left(t_{i}\right)=\left[\begin{array}{ll}
\cos \left(\omega t_{i}\right) & -\sin \left(\omega t_{i}\right)
\end{array}\right] \\
& x\left(t_{i}\right)=\left[\begin{array}{ll}
E_{d}\left(t_{i}\right) & E_{q}\left(t_{i}\right)
\end{array}\right]^{T} \\
& y\left(t_{i}\right)=E\left(t_{i}\right)
\end{aligned}
$$

The cost function to minimize:

$$
J\left[x\left(t_{i}\right)\right]=\sum_{j=0}^{i} \lambda^{i-j}\left(y\left(t_{j}\right)-H\left(t_{j}\right) x\left(t_{j}\right)\right)^{2}
$$

Where $\lambda \in(0,1)$. The cost function is minimized by WLSE method.

Figure 7 shows the flowchart of the algorithm. Figure 8 shows the temporal evolution of phase-angle of a voltage dip signal by means of the algorithm explained.

\section{2) Method of crossing by zero.}

The phase angle jump at the beginning and the end of the voltage dip are the parameters more important. So if it is not necessary to know the phase-angle at any moment it is possible to do a calculus in basis of the crossing by zero of the voltage.

In order to detect the beginning and the end of the dip it can be used a pattern signal that it is defined by the theoretical signal. This pattern signal is adjusted to real signal when there is not dip. The beginning of voltage dip is located when the real signal begins to separate of real signal and the end when they match again. Once it has been located the initial a final point it is taken the crossing by zero points before and after the real signal and pattern signal at these moments. The increments of time of these points use to calculate the phase-angle jumps. The instantaneous values are calculated adding the term wt to the initial and final angle. The points and the intervals used in the calculus are shown in figure 9.

\section{3) Parameters related to phase-angle.}

The define most interesting parameters related to phaseangle.

Border phase-angle: Angle of the wave at the moment before it is just produced an abrupt change in the instantaneous value of phase-angle signal. It is related to the position of the phasor when the instantaneous signal suffers the beginning or the end of the disturbance.
So it is considered that the signal has a module A and phase-angle $\Phi$ in the time domain. The equation before the dip is.

$$
v_{a}(t)=A \cdot \sin \left(\omega t+\phi_{a}\right)
$$

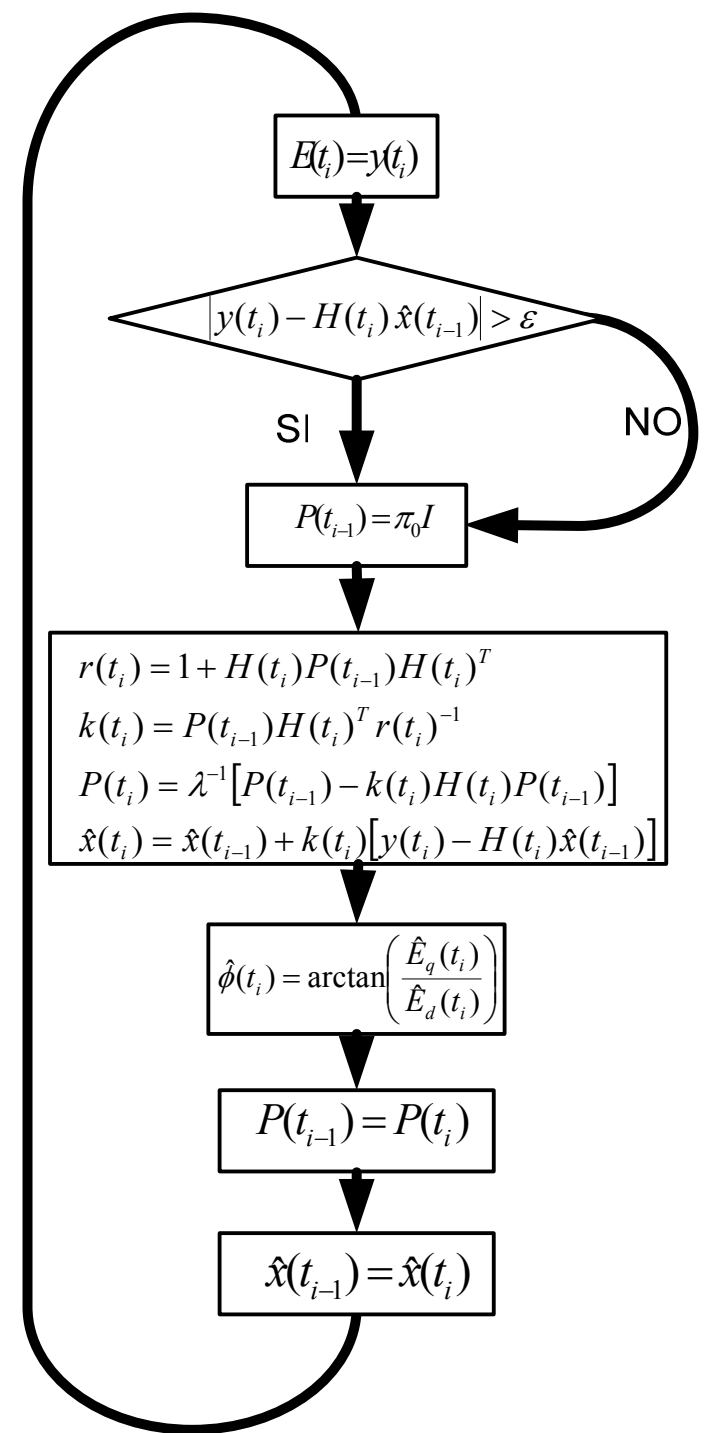

Fig. 7. Flowchart of calculus of the temporal evolution of the phase-angle.

The equation during the dip is.

$$
v_{h}(t)=H \cdot \sin \left(\omega t+\phi_{h}\right)
$$

And their respective phasors.

$$
V_{a}=A\left\lfloor\underline{\phi_{a}} \quad y \quad V_{h}=A \underline{\phi_{h}}\right.
$$

The instantaneous value of the phase-angle changes with wt. It means that the phasor pre-dip is turning at fundamental frequency before it is produced the dip. The phasor will has a phase-angle referred to $\Phi_{\mathrm{a}}$.

$$
\varphi_{f}(t)=\omega t+\phi_{a}
$$


The value of this angle has influence on instantaneous voltage jump and therefore the behaviour of the electronic machine.

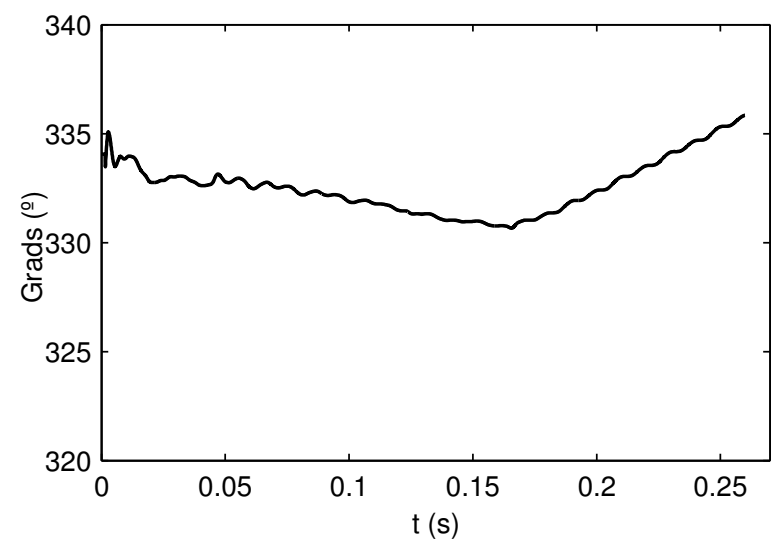

Fig. 8. Phase-angle of a real signal with a dip calculate through instantaneous phase-angle algorithm.

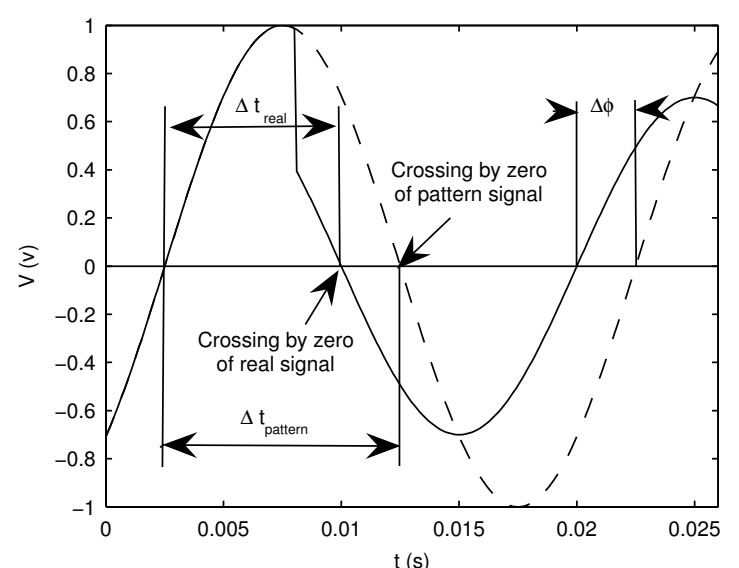

Fig. 9. Diagram of how to calculate the phase-angle jump.

Initial border phase-angle: Angle of the signal just before the dip is produced. It can be calculated with phasor or in the time domain with different physical means.

Final border phase-angle: Angle of the signal just after the dip is ended. Equal to the definition before, it can be calculated in both forms.

Phase-angle jump at time domain: It can be defined the instantaneous phase-angle jumps as the subtraction between the phase-angle of the signal a moment before and a moment after a border point in time domain. Magnitudes references $\omega t+\Phi$.

$$
\delta_{t}=\varphi_{a}-\varphi_{h}
$$

Phase-angle jump in phasors: It can be defined the frequency phase-angle jumps as the subtraction between the phase-angle of the signal a moment before and a moment after a border point working with phasors.

$$
\delta_{f}=\phi_{a}-\phi_{h}
$$

If it is substituted the expression of instantaneous phaseangle jump in the expression of frequency phase-angle jump it is.

$$
\delta_{t}=\varphi_{a}-\varphi_{h}=\left(\omega t_{a}+\phi_{a}\right)-\left(\omega t_{f}+\phi_{f}\right)
$$

It can be said that $\mathrm{t}_{\mathrm{a}} \approx \mathrm{t}_{\mathrm{f}}$. If there are no changes on the frequency during the dip, the instantaneous phase-angle jump and the phasor phase-angle jump fit.

Initial phase-angle jump: It is phase-angle jump at the moment of beginning the dip. It can be defined for both forms (phasor and time domain).

Final phase-angle jump: It is phase-angle jump at the moment of ending the dip. . It can be defined for both forms (phasor and time domain).

\section{Duration}

The duration of a dip is the difference between the final and initial moment. The detection of these moments can be done by several types of signals derived from of the instantaneous voltage wave. The measure will be different depending on that it be used.

\section{A. Used of RMS and different limits}

Any algorithm of calculus of RMS voltage can be the base to make the calculus of the duration and any of them giving acceptable results. The great problem is to define correctly the limits that mark the beginning and the end of the dip. The normative marks the beginning when the RMS voltage crosses under $0.9 \%$ of nominal voltage. The end of the dip happens when the voltage cross over this limit plus a $2 \%$ of hysteresis to avoid that the oscillations of the RMS wave give a bad measure of the duration.

\section{B. Used of Instantaneous phase-angle estimation.}

It is possible to calculate initial and final point on basis of the temporally change of the phase voltage. In order to do this, it is necessary to detect the sharp changes in that signal. When the angle jumps it used to produce a transient state. The beginning of this transient sets the initial point in the dip. After that the angle is established in a value (phase-angle during the dip) to come back to produce another transient. The end of the second transient set the final point of the dip. These phenomenons in MV are very marked and therefore it is easy to distinguish, however in LV dips is difficult to detect the beginning and end of the transients.

\section{Used of pattern wave.}

Another way to calculate the points of beginning and end it is by means of the missing voltage. This signal is equal to the rest of the pattern signal (see point Method of crossing by zero) with the actual signal. When the missing voltage signal crosses over an imposed limit, that point is the beginning of the dip. When the error signal 

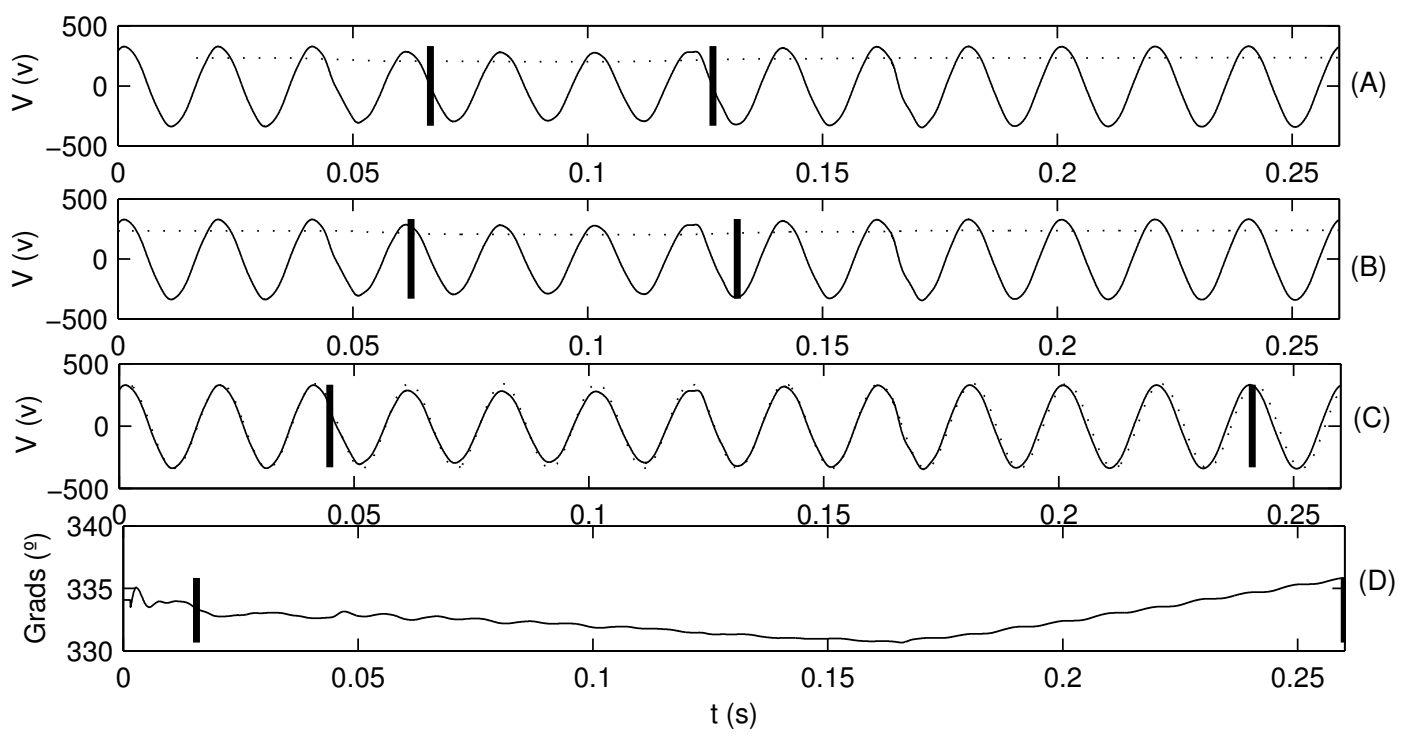

Fig. 10. Duration of a real voltage dip Signal. It has been calculated with: a) RMS wave calculated with a fixed sample window, b) RMS wave calculated with one sample mobile window, c) phase-angle signal,d) pattern signal.

comes back near initial values (It is difficult that the missing voltage come back to the exact initials conditions when the pattern signal were calculated) is when the dip is finished.

\section{Conclusions}

All the explained previously proves the complexity of the voltage dip measurement and characterization. Also, it is shown the need of measurements standardization, which has to include all the aspects of the physical phenomenon and has to be useful to the industry.

All defined parameters become complex the characterization of the dip so it is necessary to reduce the parameters to optimal minimum. The characterization according to its typology groups several of these parameters and can be considered as a final step after measuring the magnitude, the phase-angle and the duration of the dip.

There are many application fields where the measurement of voltage dips can give a better picture of electric problems. One of most trendy field is the study of connection and disconnection of renewable energy systems caused by voltage dips. The study of parameters in dips helps to understand the behaviour of electronic systems that control these systems.

\section{Referentes.}

[1] M. T. Aung and J. V. Milanovic, "The Influence of Transformer Winding Connections on the Propagation of Voltage Sags, "Power Delivery, IEEE Transactions on, vol. 21, pp. 262-269, 2006.

[2] J. Barros and R. I. Diego, "Discussion of "Sensitivity of Personal Computers to Voltage Sags and Short Interruptions", " Power Delivery, IEEE Transactions on, vol. 21, pp. 543-544, 2006.
[3] S. Z. Djokic and J. V. Milanovic, "Closure on "Sensitivity of Personal Computers to Voltage Sags and Short Interruptions", "Power Delivery, IEEE Transactions on, vol. 21, pp. 544-545, 2006.

[4] A. Moreno-Munoz and J. J. G. de la Rosa, "Analysis of voltage dips in PWM AC-DC converters, "Power Electronics, Electrical Drives, Automation and Motion, 2006. SPEEDAM 2006. International Symposium on, pp. 191-194, 2006.

[5] G. Carpinelli, C. Di Perna, P. Caramia, P. Varilone, P. Verde, R. Chiumeo, I. Mastandrea and F. Tarsia, "On robustness of distribution systems against voltage dips, "Power Electronics, Electrical Drives, Automation and Motion, 2006. SPEEDAM 2006. International Symposium on, pp. 1310-1315, 2006.

[6] J. A. Martinez and J. Martin-Arnedo, "Voltage Sag Studies in Distribution Networks-Part I: System Modeling," Power Delivery, IEEE Transactions on, vol. 21, pp. 1670-1678, 2006.

[7] G. Olguin, F. Vuinovich and M. H. J. Bollen, "An optimal monitoring program for obtaining Voltage sag system indexes, "Power Systems, IEEE Transactions on, vol. 21, pp. 378-384, 2006.

[8] J. Pedra, L. Sainz, F. Corcoles and L. Guasch, "Symmetrical and unsymmetrical voltage sag effects on three-phase transformers," Power Delivery, IEEE Transactions on, vol. 20, pp. 1683-1691, 2005.

[9] Y. Chen, L. Sanchez, K. Y. Smedley and G. Chen, "One-Cycle Controlled Unified Power Quality Conditioner For Load Side Voltage Sag Compensation," Power Electronics Speciaselections, 2005 IEEE 36th Conference on, pp. 282-288.

[10] R. de Andrade Jr, A. C. Ferreira, G. G. Sotelo, J. L. S. Neto, L. G. B. Rolim, W. I. Suemitsu, M. F. Bessa, R. M. Stephan and R. Nicolsky, "Voltage sags compensation using a superconducting flywheel energy storage system, "Applied Superconductivity, IEEE Transactions on, vol. 15, pp. 2265-2268, 2005.

[11] J. V. Milanovic and C. P. Gupta, "Probabiselectionic assessment of financial losses due to interruptions and voltage sags - part II: practical implementation, "Power Delivery, IEEE Transactions on, vol. 21, pp. 925-932, 2006.

[12] E. EspinosaJuarez and A. Hernandez, "An Analytical Approach for Stochastic Assessment of Balanced and 
Unbalanced Voltage Sags in Large Systems," Power Delivery, IEEE Transactions on, vol. 21, pp. 14931500, 2006.

[13] J. C. Gomez and M. M. Morcos, "Coordination of voltage sag and overcurrent protection in DG systems, " Power Delivery, IEEE Transactions on, vol. 20, pp. 214-218, 2005.

[14] Z. F. Hussien, A. B. Ismail, W. C. Lee, A. M. Busrah and M. F. M. Siam, "Voltage Sag Mitigation using NAS Battery-based Standby Power Supply, "Power Electronics and Drives Systems, 2005. PEDS 2005. International Conference on, vol. 2, pp. 1317-1321, 2005.

[15] M. H. J. Bollen, "Algorithms for characterizing measured three-phase unbalanced voltage dips, "Power Delivery, IEEE Transactions on, vol. 18, pp. 937-944, 2003.

[16] M. H. J. Bollen, "Voltage sags in three-phase systems, "Power Engineering Review, IEEE, vol. 21, pp. 8-11, 15, 2001.

[17] M. H. J. Bollen and E. Styvaktakis, "Characterization of three-phase unbalanced dips (as easy as one-twothree?), "Power Engineering Society Summer Meeting, 2000. IEEE, vol. 2, pp. 899-904 vol. 2, 2000.

[18] M. H. J. Bollen, E. Styvaktakis and I. Y. H. Gu, "Analysis of voltage dips for event identification, "Power Quality: Monitoring and Solutions (Ref. no. 2000/136), IEE Seminar on, pp. 0_7-4/4, 2000.

[19] Lidong Zhan and M. H. J. Bollen, "Characteristic of voltage dips (sags) in power systems, "Power Delivery, IEEE Transactions on, vol. 15, pp. 827-832, 2000.

[20] M. Ohrstrom and L. Soder, "A comparison of two methods used for voltage dip characterization, "Power Tech Conference Proceedings, 2003 IEEE Bologna, vol. 4, pp. 6 pp. Vol., 2003."

[21] Electromagnetic compatibility (EMC), Part 4-34: Testing and measurement techniques - Voltage dips, short interruptions and voltage variations immunity tests for equipment with input current more than $16 \mathrm{~A}$ per phase." CEI/IEC 6100-4-34:2005.

[22] Mañana M. "Aportaciones a la representación, detección y clasificación de perturbaciones conducidas en sistemas eléctricos de potencia". Tesis doctoral. Mayo 2000. Universidad de Santander.

[23] H. S. Song, K. Nam and P. Mutschler, "Very Fast Phase Angle Estimation Algorithm for a Single-Phase System having Sudden Phase Angle Jumps,"

[24] H. S. Song and K. Nam, "Instantaneous phase-angle estimation algorithm under unbalanced voltage-sag conditions, "Generation, Transmission and Distribution, IEE Proceedings-, vol. 147, pp. 409-415, 2000. 